\title{
Internacionalización de emprendimientos culturales de Latinoamérica
}

\section{Internationalization of cultural enterprises in Latin America}

Andrea Carolina Redondo Méndez ${ }^{1}$, Carlos Andrés Pinzón Muñoz ${ }^{2}$, Oswaldo Ospina Martínez ${ }^{3}$

INFORMACIÓN DEL

\section{ARTÍCULO}

Fecha de recepción: 23 de Julio de 2020.

Fecha de aceptación: 6 de Noviembre de 2020.

1 Magíster en Investigación Social Interdisciplinar, Universidad Distrital Francisco José de Caldas. Docente-investigadora, Universitaria Agustiniana-Colombia

E-mail:

andrea.redondo@uniagustiniana.edu.co Código ORCID:

https://orcid.org/0000-0001-9226-8626

2 Magíster en Desarrollo Sostenible y Medio Ambiente, Universidad de Manizales. Docente-investigador, Universitaria Agustiniana-Colombia. E-mail:

carlos.pinzonm@uniagustiniana.edu.co Código ORCID

https://orcid.org/0000-0001-8376-0504

${ }^{3}$ Maestría en Psicología del Consumidor, Fundación Universitaria Konrad Lorenz. Docente-investigador, Universitaria Agustiniana-Colombia.

E-mail:

oswaldo.ospinam@uniagustiniana.edu.co Código ORCID

https://orcid.org/0000-0003-3889-5973

CITACIÓN: Redondo Méndez, A.C., Pinzón Muñoz, C.A., \& Ospina Martínez, O. M.(2020). Internacionalización de emprendimientos culturales de Latinoamérica. Podium, 38, 87-104. doi:10.31095/podium.2020.38.6

ENLACE DOI:

http://dx.doi.org/10.31095/podium.202 0.38 .6

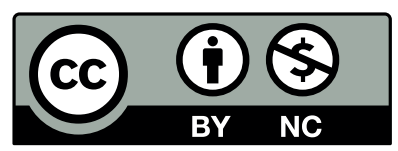

\section{Resumen}

El objetivo de la investigación es identificar las estrategias de cooperación de los emprendimientos culturales en Latinoamérica que permiten su internacionalización. Tuvo un enfoque descriptivo correlacional con un diseño metodológico cuantitativo y no experimental que facilitó la identificación del pensamiento sistémico como punto de partida para el desarrollo del trabajo colaborativo dentro del sector cultural, mediante una encuesta a 450 empresarios pertenecientes a las Industrias Culturales Latinoamericanas. Como principales hallazgos y conclusiones se logró la identificación de las prioridades en torno a la aplicabilidad y reconocimiento del emprendimiento, evidenciando que quienes han logrado su internacionalización han utilizado alianzas estratégicas o han llegado a mercados externos mediante una financiación propia. Así también, se demostró que tanto el trabajo en redes como la cooperación transfronteriza son las estrategias principales para lograr sus procesos de internacionalización debido a la falta de apoyos gubernamentales.

\section{Palabras Clave:}

Internacionalización, emprendimiento creativo, redes, cooperación, estrategias, cultura.

Clasificación JEL: Z10, Z13, D21.

\begin{abstract}
This research purports to identify cultural entrepreneurship's cooperation strategies in Latin America; such strategies permit entrepreneurship's internationalization. The research had a descriptive and correlational focus and had a quantitative and non-experimental methodological design. This design facilitated identification of systemic thought as a starting point for the development of collaborative work within the cultural sector. Four hundred and fifty businessmen were surveyed; they belonged to Latin-American Cultural Industries. The main finding and conclusion was the achievement of priority identification in connection with entrepreneurship's applicability and reconnaissance. It became evident that those who achieved internationalization did so either by using strategic alliances or by reaching foreign markets by means of their own financing. Lack of government support caused both networking and cross-border cooperation to be the main strategies to achieve entrepreneurship's internationalization.
\end{abstract}

Keywords:

Internationalization, creative entrepreneurship, networks, cooperation, strategies, culture.

JEL Classification: Z10, Z13, D21.

\section{7}

PODIUM No. 38, Diciembre 2020, pp. 87-104

(C) Universidad Espíritu Santo - UEES

ISSN: 1390-5473 e-ISSN: 2588-0969 


\section{Introducción}

Las industrias culturales y creativas se reconocen, a nivel mundial, como aquellos sectores de actividad organizada que tienen como objeto principal la producción o la reproducción, la promoción, la difusión y/o la comercialización de bienes, servicios y actividades de contenido cultural, artístico o patrimonial (UNESCO, 2009). A nivel latinoamericano, participan con el $6 \%$ de los ingresos totales de las industrias en el mundo y con el $7 \%$ de los empleos, siendo parte de un sector en crecimiento, con opciones y con espacios de integración de la población civil.

Las industrias culturales y creativas en Latinoamérica han permitido el desarrollo de iniciativas empresariales, que no solo se caracterizan por ser innovadoras sino porque vinculan espacios sociales y económicos que están en una constante búsqueda de espacios de reconocimiento y difusión, debido a la multiplicidad de subsectores que vincula y a su vez debido a las actividades que integra. Esto es, actividades culturales propias del ser humano, talentos $\mathrm{y}$ habilidades que se empiezan a desarrollar desde rasgos identitarios particulares y que no requieren, necesariamente, de formación o instrucción para ser reconocidas. Dentro del mercado internacional, la cultura está generando la apertura hacia nuevos espacios de difusión de su producción y nuevos escenarios de consumo por parte de más población, ya que emerge de las fortalezas locales y de su propia identidad (Hernández-Acosta, Redondo, y Ospina, 2018).
El objetivo de la investigación fue identificar las estrategias de cooperación del sector cultural latinoamericano, que han permitido la materialización de procesos de internacionalización de sus emprendimientos culturales, bajo el marco de la economía creativa, creada y desarrollada por Howkins (2001), para quien la creatividad, como la creadora de un sentido de identidad es, además, una forma de hacer negocios y brindarle una dimensión económica.

Así, este artículo presenta en primer lugar, una revisión de literatura enfocada a la creatividad, las industrias culturales y creativas, los modelos de internacionalización para el sector cultural, la teoría de redes y su relación con el networking cultural. Luego explica la metodología de la aplicación del instrumento, así como las variables seleccionadas además de la competencia seleccionada para este caso, el pensamiento sistémico; para, finalmente, presentar los resultados de la investigación.

\section{Revisión de literatura}

\section{Emprendimientos: industrias culturales en movimiento}

Las industrias culturales y creativas (ICC) vinculan sectores tradicionales y modernos que incluyen una serie de actividades que poseen todo un andamiaje académico y formativo (Ver Tabla 1). Se han convertido en un sector que incide en el desarrollo socio-económico de un país en tanto va generando ingresos y empleos, basados en las habilidades del ser humano, por lo 
que generan unos ingresos de US\$124.000 millones (el 6\% del mercado total de las ICC) y 1,9 millones de empleos -el 7\% de los empleos totales de las ICC- (CISAC, 2015). Utilizan la creatividad como estructura de su producción y se presentan, tanto como generadoras de riqueza, como creadoras de valor de identidad, mostrando su propiedad económica y su carácter cultural. Así, presentan productos culturales y creativos que están cargados de valor agregado debido a su carácter comunicativo y diferencial. En otras palabras, los variados tipos de actividades culturales y los bienes y servicios que estas actividades producen, son evaluados por quienes los hacen y los compran, ya sea por razones sociales y culturales que posiblemente sobrepasan una simple valoración económica (PNUD, 2010).

Tabla 1 .

Industrias culturales y creativas

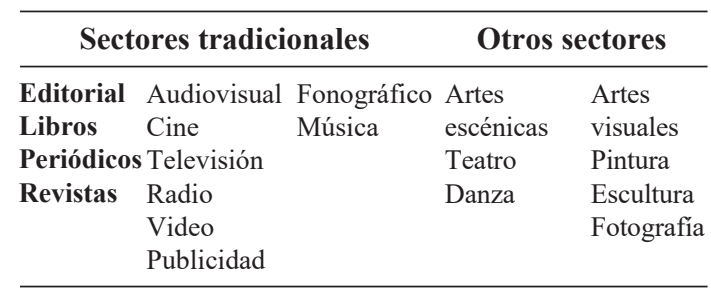

Fuente: Elaboración propia a partir de UNESCO (2009).

El panorama latinoamericano frente al sector cultural manifiesta un crecimiento desde la formalización y el impulso de los sub-sectores culturales, de manera que emergen actividades rentables en un mercado ávido de posibilidades y que se posicionan como garantes de desarrollo social y de generación de espacios de ocupación para la sociedad civil. Este sector, se encuentra como punto prioritario de las agendas de los países latinoamericanos, siendo fundamental su regulación y su construcción de datos, que permitan claras y definidas caracterizaciones, diagnósticos y mapeos, además del desarrollo de normatividad e institucionalidad propia (Hernández-Acosta, 2020). Y es que, "las industrias culturales y creativas han logrado posicionarse como un elemento de desarrollo para los países que han incursionado y apoyado las iniciativas de este sector, siendo representativa la generación de emprendimientos y la innovación en sus prácticas. Desarrollando un lugar importante para las investigaciones $\mathrm{y}$ procesos de innovación, que, desde la academia, se logran forjar" (Rodríguez, Redondo, Ospina, y Pinzón, 2020, pp. 171).

Ahora bien, la noción de emprendimiento creativo hace referencia al conjunto de prácticas, habilidades y herramientas de empresas y organizaciones culturales respecto a su desempeño y alcance, en donde, se busca un rendimiento positivo que vincule su producción artística, si se quiere, con el contexto que lo rodea. Los emprendimientos creativos, como iniciativas empresariales, hacen uso del potencial de las industrias culturales y creativas para poner en marcha planes y estrategias de mercado con el fin de lograr su inserción en el contexto internacional. Lo que las sitúa dentro de los sectores más dinámicos del sistema internacional y uno de los que mayor perspectiva de crecimiento económico poseen. Así, "se trata de una combinación de elementos de marca, mercadeo, finanzas, recursos humanos, gerencia de 
proyectos y muchas otras herramientas que buscan generar riqueza, pero también impactar en el entorno local" (Hernández-Acosta, 2020, p. 10).

En Latinoamérica, este tipo de industrias se ha caracterizado por su aporte y desarrollo económico, generando una importante participación dentro del PIB $-6 \%$ de la región(Rodríguez, 2018), y se ha establecido como uno de los motores de crecimiento de cada uno de los países latinoamericanos, debido a su constante transformación en términos organizativos, alejándose de paradigmas tradicionales que han llevado a una integración económica que permite la expansión a mercados internacionales, pero que, sin embargo, no han logrado los resultados esperados en términos de impacto real, debido al poco conocimiento de estrategias de internacionalización por parte de los empresarios creativos, y debido a la falta de apoyo gubernamental.

El desarrollo de empresas culturales potencia las particularidades del sector al enfocarse en los elementos diferenciadores tanto en bienes como en servicios, que, en este caso, parten de la propia identidad al hacer uso del talento, la creatividad y la innovación como parte de sus cadenas productivas y de valor, al poseer procesos de producción, circulación y apropiación social, los cuales son fijados sobre soportes tangibles o electrónicos, con circulación masiva. Además, frente a los lugares de integración y producción, conforman identidades y promoción de ciudadanía bajo imaginarios sociales, que se encuentran articulados con las lógicas de los mercados y leyes de comercialización (SIPPE, 2013). El desarrollo de este tipo de iniciativas creativas supone el establecimiento de 3 elementos fundamentales para su apropiación y efectividad, todo dentro de parámetros de búsqueda de estrategias de cooperación, a saber, la ciudadanía cultural, entendida como la integración e interdependencia de la sociedad respecto a los proyectos que vinculan la identidad y el desarrollo de las habilidades humanas; el rendimiento cultural, que propone un trabajo en redes desde todas las esferas de la sociedad; y la gerencia cultural, que le brinda al sector una formalidad, necesaria, para emprender y lograr mayor competitividad (Hernández-Acosta, 2020).

\section{Creatividad y cultura: su reconocimiento}

Según la CISAC (2015), el uso del contenido inmaterial, por parte de las industrias culturales, es el punto de partida para la creación de valor y de reconocimiento, debido a que permite la apropiación de características innatas y únicas de su identidad que, además, se muestran en un mercado internacional carente de la multiplicidad real de las identidades de cada territorio, generando así, en la sociedad, la identificación y posicionamiento como protagonistas directos del patrimonio cultural de cada nación.

Las creaciones de la mente son un activo que hace parte del patrimonio de la empresa y como tal, valen y pueden 
protegerse $\mathrm{y}$, lo más importante, usarse para sacar un beneficio. Y no solo se trata del valor monetario que se pueda extraer, sino que a través del uso de competencias emprendedoras que logran entender la realidad desde diversos puntos de vista que permiten el posicionamiento del bien o del servicio cultural como garante de identidad social, logra situarlo como parte de la especificidad de la coyuntura social, pero una coyuntura, en este caso, atemporal, debido a que permite vislumbrar elementos históricos a través del arte contemporáneo de su transformación, y que aun así logra demostrar la identidad. El concepto de creatividad, se ha convertido en uno de los procesos generadores de ideas cargadas de valor para el sector cultural, y que proyecta o permite el acercamiento a procesos de innovación que son relevantes en la producción de las industrias culturales y creativas. Asimismo, por medio de la creatividad se logra responder a situaciones en donde no se trata de novedad sino de conexión de ideas alejadas, con el fin de dar respuestas prácticas frente a situaciones que presentan escasez de algún tipo de recurso (Howkins, 2001). Es decir, sin necesidad de acudir a la búsqueda de recursos económicos, materias primas $\mathrm{o}$ insumos en general de altos costo y difícil acceso, se recurre a la propia capacidad del representante cultural para crear a través, tanto de la preservación identitaria como de la consecución de materia intangible e inmaterial, posicionando al sector, o la empresa como ente reconocido socialmente de preservación y exaltación de la identidad cultural. Uno de los ejemplos más representativos, sobre el uso de la creatividad y su relación con las industrias culturales, tiene que ver con las configuraciones narrativas y la producción de contenido audiovisual que actualmente se genera, demostrando la influencia y el alcance que se logra dar a partir de creaciones, ya sean infantiles o para público adulto. De tal manera que, se logra la creación de personajes con alto contenido cultural propicios para emitir mensajes particulares, usando la creatividad como mecanismo (Ortega-Mohedano, JiménezSánchez, y Lavín, 2018).

\section{Economía creativa: John Howkins}

La noción de economía creativa, se debe al profesor británico John Howkins (2001), quien manifiesta que se trata de una nueva forma de pensar y de hacer, basándose en características subjetivas del ser humano y aprovechando los procesos colectivos de innovación con el fin de generar negocio. Son conceptos que no van separados, y que representan en esta investigación el espacio adecuado para su análisis, teniendo en cuenta que se habla de espacios en donde se reúnen expertos creativos para hacer negocio. Así, se crea un nuevo paradigma económico que explica la generación de riqueza a partir de las especificidades de las actividades y relaciones para crear valor $\mathrm{y}$, en mayor medida, la forma de proteger estas creaciones derivadas, en términos de la propiedad intelectual, entonces, es atrayente destacar que en la nueva economía el tamaño de los países y de las regiones es menos importante. En esta economía el tamaño no es lo determinante, sino que es la rapidez con 
que uno se mueve y la capacidad de las personas (Saucedo, 2020). Entonces, la producción creativa es un garante de reconocimiento social nacional $\mathrm{e}$ internacional, en tanto materializa las ideas, entendidas como las iniciativas de uso de elementos característicos de identidad, a través del uso de las herramientas enfocadas a la identificación de elementos inmateriales y de gran valor histórico para una sociedad colmada de elementos propios de su patrimonio y ávidos por difusión. Situando estos, en lugares privilegiados de conocimiento y consumo, precisamente por su presentación a través de la inteligencia social al utilizar la interconexión de la comunicación en beneficio de su elaboración.

\section{Internacionalización de la cultura}

La cultura es una aglomeración de prácticas tradicionales mediante habilidades propias del ser humano, que representan la identidad de un espacio determinado y la riqueza de un pueblo; por esto, cuando se habla de su internacionalización no solo se menciona como generación de riqueza sino como eliminación de barreras territoriales para el conocimiento y la apropiación de prácticas representativas que tienden al respeto por la diversidad. La existencia de emprendimientos creativos con proyecciones y prácticas de internacionalización logra beneficiar el sector cultural en tanto, y siguiendo las dimensiones planteadas por el Ministerio de Cultura de Colombia (2010) permite actuar sobre cinco aspectos relevantes:

1. Fortalecimiento de las experticias por medio de intercambios culturales, sean académicos o prácticos, lo que generaría mayor reconocimiento y experiencia.

2. Circulación de contenidos, por medio de la participación en eventos internacionalmente reconocidos con fines de visibilización y consolidación de procesos culturales

3. Emprendimiento cultural, para la financiación y apoyo general para iniciativas culturales que puedan fortalecer el sector mediante alianzas externas.

4. Movilidad de artistas, para mayores niveles de reconocimiento internacional sobre las prácticas culturales de cada país.

5. Dotación, mediante convenios internacionales que generen donaciones para el sector cultural.

Es importante tener en cuenta que la cultura hace parte importante de los elementos que componen la política exterior de los estados modernos, sabiendo que determina su emerger a partir de la proyección internacional que se hace de ella; es decir, dentro de las relaciones internacionales, la cultura es un componente necesario para la transformación, si se quiere, de las actividades económicas y políticas que determinan los acercamientos entre estados, lo que supone un lugar privilegiado $\mathrm{y}$, además, necesario del conocimiento de las mismas y de su proyección internacional (Ramírez, 2013). 
La teoría de redes y el networking cultural

El planteamiento de la internacionalización de las prácticas culturales mediante la representatividad de los emprendimientos creativos se ha visto reflejada en la teoría de redes, cuya base estructural es el desarrollo de organizaciones y empresas a través de relaciones sociales para la consecución de información sobre mercados externos.

Las redes de negocio permiten una mayor interacción entre diversos actores, a saber, clientes, distribuidores, competidores y gobierno. Y se refieren a un conjunto de redes de trabajo que va a determinar los niveles de internacionalización y su conocimiento de los mercados (ver Tabla 2).

Tabla 2.

Modelo de redes de Johanson y Mattson

\begin{tabular}{cccc}
\hline \multicolumn{3}{c}{ Grado de internacionalización de la red } \\
\hline & Bajo & $\begin{array}{c}\text { Bajo } \\
\text { Empresa } \\
\text { iniciadora }\end{array}$ & $\begin{array}{c}\text { Alto } \\
\text { Empresa } \\
\text { rezagada }\end{array}$ \\
$\begin{array}{c}\text { internacionalización } \\
\text { de la empresa }\end{array}$ & Alto & $\begin{array}{c}\text { Empresa } \\
\text { internacional } \\
\text { en solitario }\end{array}$ & $\begin{array}{c}\text { Empresa } \\
\text { internacional } \\
\text { junto con } \\
\text { otras empresas }\end{array}$
\end{tabular}

Fuente: Elaboración propia a partir de Johanson,y Mattson. (2015).

Desde la teoría de redes, el networking se ha convertido en una de las estrategias más importantes para el sector cultural, debido a que, mediante las múltiples interacciones, logra el contacto con socios estratégicos que permiten el ingreso a mercados internacionales para empresas que, sin embargo, no poseen experiencia suficiente en nuevos mercados.
Las actividades en la red le permiten a la empresa mantener relaciones que le ayudarán a tener acceso a recursos y mercados. Una presunción básica de este modelo es que las empresas necesitan recursos controlados por otras empresas, los cuales pueden ser obtenidos debido a su posición dentro de la red (Cardozo, Chavarro, y Ramírez, 2007, p.14).

Así, el mecanismo de inserción a mercados internacionales por parte de las industrias culturales y creativas ha sido el networking cultural de manera formal e informal, debido a las posibilidades que le brinda al sector, al establecerse como mecanismo para conseguir alianzas estratégicas y posibilidades de difusión de sus actividades.

El trabajo en redes permite el intercambio de información, contactos y espacios con personas, organizaciones o hasta gobiernos con intereses comunes y es el mecanismo de mayor preferencia para el sector cultural, respecto a la búsqueda de espacios laborales y de difusión de las prácticas culturales. Esto es, como estrategia, brinda la posibilidad a empresas y emprendimientos de cualquier tamaño y con variedad de experiencias, la posibilidad de ingresar a mercados internacionales (Lindsay, 2005).

\section{Internacionalización desde la cooperación transfronteriza}

Existe un componente de cooperación, para el caso latinoamericano, en tanto las 
estrategias a utilizar representan un desarrollo tanto de empresa como de Estado al momento de entablar relaciones sociales y económicas que permitan la exploración y posicionamiento en mercados internacionales. Esto es, el fortalecimiento de Latinoamérica como región va a permitir mejores resultados para procesos de internacionalización del sector cultural en la medida en que se forje una unión transfronteriza (Hernández-Acosta, 2020).

Se debe tener en cuenta que a partir de la década de los 90's aparece un nuevo discurso en Latinoamérica, respecto a la necesidad de vincular la cultura a procesos de desarrollo y procesos de crecimiento económico, si bien por presiones sociales o por innovar con temas culturales en procesos de integración económica, lo que supuso el establecimiento relevante de estándares de protección de diversidad cultural. Si bien, el reconocimiento que los gobiernos latinoamericanos le han otorgado a la elaboración de políticas en torno a la cultura no ha sido replicado en todos los estados de la región, sí existe un conjunto de normatividad y jurisprudencia para casos específicos, que vale la pena tomar como referentes de futuras cooperaciones (Yúdice, 2019).

El Acuerdo General sobre el Comercio de Servicios (AGCS) establece excepciones de estatus jurídico especial para la industria audiovisual en términos de la aplicación de la cláusula de nación más favorecida Entre los países que establecieron esa excepción se encuentran algunos países latinoamericanos: Bolivia,
Brasil, Chile, Colombia, Ecuador y Venezuela. Lo anterior se debió a la existencia de normatividad adicional frente al tema de industria cultural, es decir, el Convenio de Integración Cinematográfica Iberoamericana firmado el 11 de noviembre de 1989 por Argentina, Bolivia, Brasil, Colombia, Cuba, Ecuador, España, Perú, México, Nicaragua, Panamá, República Dominicana y Venezuela; y el Acuerdo Latinoamericano de Cooperación Cinematográfica de 1989 suscrito por Argentina, Brasil, Colombia, Cuba, Ecuador, México, Nicaragua, Panamá, Perú, República Dominicana y Venezuela. La respuesta generada desde el AGCS mostró una especie de victoria parcial para los defensores de la cultura ya que consiguieron: "un tratamiento especial y limitado en el terreno de la producción audiovisual [por lo cual] los norteamericanos- detrás de las multinacionales-continúan acosándolos" (Ghalioun, 1998, p.3).

El MERCOSUR (1996), y uno de sus protocolos, el Protocolo de integración cultural (aprobado mediante Decisión No.11/96 del Consejo del Mercado Común), no abarca la totalidad de aspectos vinculantes, pero sí presenta una postura frente al patrimonio cultural, pero no hace referencia directa al tema. En este mismo sentido, el MERCOSUR cultural se reunió, por primera vez en Brasil, los días 2 al 4 de febrero de 1996 y decidió crear una Reunión de Legisladores de las comisiones de cultura de los parlamentos de los cuatros países miembros, con el fin de integrar esfuerzos con los ministros de cultura de cada país y crean el Parlamento 
Cultural del MERCOSUR (PARCUM) que es una entidad que se compone por unas comisiones de trabajo, dentro de las cuales se incluyó la comisión de industrias culturales, que se encarga de crear los protocolos sobre patrimonio cultural, intercambio y capacitación de recursos humanos, circulación de personas y bienes culturales, telemática y legislación, y en crear un programa para entrenar especialistas en gestión cultura.

Y, la Comunidad Andina creó, mediante la Decisión 760 (Consejo Andino de Ministros de Relaciones Exteriores, 2011) el Consejo Andino de Ministros de Cultura y Culturas y como una de sus funciones se estableció el formular propuestas de estrategias subregionales para el desarrollo y fomento de las industrias culturales en el marco de un Plan Andino de Industrias Culturales y crear el Comité Andino de Industrias Culturales. En este mismo sentido se han llevado a cabo los siguientes programas referentes a la protección y posicionamiento cultural:

- Programa de Armonización de Estadísticas y Cuentas Satélite de la Cultura.- Mediante la Decisión 782, del 11 de marzo de 2013, que generó el "Programa de Armonización de Estadísticas y Cuentas Satélite de la Cultura" cuyo objetivo fue medir, mediante un modelo homogenizado, los insumos utilizados y los aportes realizado por el sector cultural a la economía nacional y regional. Desde abril de 2013, se realizan, mensualmente reuniones del Grupo de Expertos Gubernamentales de los Países Miembros, para avanzar conjuntamente en la elaboración y levantamiento de la información.

- Portal Cultural de la Comunidad Andina (CULTURANDE, 2011). En donde los países de la CAN se comprometieron a fortalecer este portal mediante los programas y acciones de los Comités Andinos de Industrias Culturales, Patrimonio Cultural Material e Inmaterial y Lucha contra el tráfico ilícitos de bienes culturales. Se trata entonces de una plataforma cultural que pretende constituirse en una extensión de los Sistemas de Información cultural de los Ministerios de Cultura, con el fin de difundir la cultura andina en el mundo.

Todos estos programas manifiestan el interés vinculante actual sobre el sector cultural latinoamericano y la necesaria cooperación transfronteriza con fines de internacionalización, basados en la teoría de redes y el tamaño que pueda adquirir, debido a que "es evidente que las grandes empresas diversificadas tienen más oportunidades de explotar los beneficios gracias a los lazos establecidos a través de sus redes" (Cardozo, et al. 2007, p. 13) $\mathrm{y}$ es mediante este tipo de iniciativas legislativas y de apoyo estatal que las industrias culturales y creativas logran una inserción en nuevos mercados.

\section{Metodología}

La investigación se desarrolló en dos fases, la primera fue exploratoria y se enfocó en la utilización de diversas fuentes secundarias de información que permitieron una revisión bibliográfica del tema de estudio para la construcción de 
antecedentes y marco teórico. La segunda fase comprendió un estudio descriptivo correlacional transversal (Hernández, Fernández y Baptista, 2014) a través del método de encuesta a una muestra no probabilística de 450 empresarios culturales y artistas de diez países: Argentina, Bolivia, Brasil, Chile, Colombia, Ecuador, Paraguay, Perú, Uruguay y Venezuela, pertenecientes a los subsectores de: artes escénicas, audiovisual, diseño, editorial, música, animación y videojuegos, que participaron en el evento MICSUR, en su edición 2016, realizado en la ciudad de Bogotá.

Este estudio transversal permitió describir los aspectos relevantes de las competencias de un empresario creativo y como investigación correlacional tuvo como propósito mostrar o examinar la relación entre variables o resultados de variables, pero en ningún momento explicó que una sea la causa de la otra. "La correlación examina asociaciones, pero no relaciones causales, donde un cambio en un factor influye directamente en un cambio en otro" (Bernal, 2010, p.114). En este caso se trata de las variables pensamiento sistémico e internacionalización.

Para conocer el comportamiento de las variables de interés, se diseñó un cuestionario estructurado, a partir de investigaciones previas, buscando el reconocimiento de las variables en estudio. Se diseñaron veinte y cuatro (24) preguntas, las cuales se dividieron en 6 temáticas, a saber, las competencias caracterizan a un emprendedor creativo como lo son: Comunicación Asertiva
(CA), Amplitud Perceptual (AP), Creatividad e Innovación (CI), Inteligencia Social (IS), Orientación al Logro (OL) y Pensamiento Sistémico (PS). Este instrumento fue validado a través de un panel de expertos latinoamericanos en el sector cultural y para esta fase de investigación sólo se utilizó la variable PS como eje de análisis a través de sus descriptores: Acceso a auxilios y beneficios, relación de sus trabajadores con su preparación profesional, procesos innovadores, conocimiento del mercado, reconocimiento de grupos artísticos, superación de estándares de desempeño y la evaluación de objetivos; con el propósito de identificar las estrategias encaminadas a la internacionalización de sus actividades culturales. Para el análisis estadístico de los datos se utilizó el software SPSS 18.

\section{Resultados}

De las estrategias utilizadas por las empresas culturales (ver Figura 1), el 63,4\% busca la satisfacción al cliente, el 39,2\% realiza alianzas estratégicas, el $37,7 \%$ busca consultoría o asesorías, el $27,8 \%$ utiliza investigación de mercados y 27,3\% realiza benchmarking dentro de su sector.

El 55,3\% de los encuestados conoce

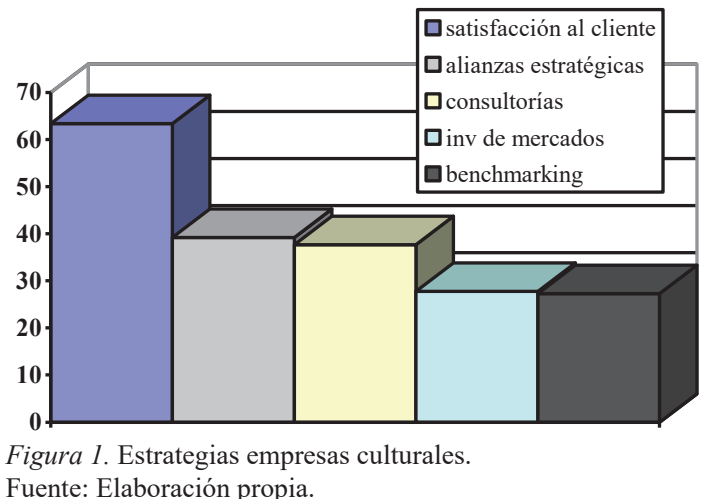


algún mecanismo del gobierno para proteger la industria nacional, los cuales son en su gran mayoría legislación referente a incentivos para diversos sectores de la economía cultural; el grado de asociación es relativamente bajo ya que solo el $42,5 \%$ pertenece a una asociación o agremiación, sin embargo, no señalan de manera puntual a qué agremiación o asociación pertenecen.

El $80,5 \%$ utiliza recursos propios para el desarrollo de su actividad, el 32,9\% recurre a inversores, un $24,6 \%$ a entidades financieros y el $23,5 \%$ a auxilios del Estado. Los márgenes de ganancia frente a la inversión que realizan se encuentran entre un 0 al 20\%, el $22,4 \%$, entre 21 al $40 \%$, el $34,2 \%$, entre 41 al $60 \%$, el $27,6 \%$ y más del $61 \%$ el 15,8\%.

\section{Análisis de Correlaciones}

Esta investigación correlacional se enfocó en establecer la relación lineal existente entre el cambio de performance para conseguir mayores oportunidades en el mercado internacional (Cambio performance MI) y los descriptores de la variable PS: Beneficios o auxilios estatales o privados, la razón de ser de los cargos del equipo de trabajo, la utilización de nuevas formas o nuevos elementos para mejorar o cambiar sus presentaciones, la comprensión de la visión y expectativas del mercado, el reconocimiento de la contribución de otros grupos artísticos, y la orientación de acciones llevadas a cabo para lograr y superar los estándares de desempeño y los plazos fijados (ver Tabla 3). Se encontró la existencia de relaciones lineales positivas estadísticamente significativas.

En consecuencia, se logra dar respuesta a la pregunta que guió esta investigación, respecto al reconocimiento de las estrategias de cooperación de los emprendimientos culturales que los lleven a la internacionalización, logrando verificar que se configura desde el trabajo en redes, debido a que es la manera más eficaz hasta el momento, para el sector cultural, de conocer y acceder a mercados internacionales y de poder establecer alianzas estratégicas importantes para su iniciativa. La relación con uso del pensamiento sistémico se refleja en el reconocimiento que tienen los encuestados del ecosistema creativo al cual pertenecen y de la participación de artistas y creadores de diferentes partes de la región en un mismo espacio de

Tabla 3.

Matriz de Correlaciones del Cambio de Performance MI con las variables descriptoras del Pensamiento Sistémico

\begin{tabular}{ccccccccc}
\hline & & $\begin{array}{c}\text { Cambio } \\
\text { performance } \\
\text { MI }\end{array}$ & Subsidios & $\begin{array}{c}\text { Razón } \\
\text { de Ser } \\
\text { Cargos }\end{array}$ & $\begin{array}{c}\text { Mejora de } \\
\text { Presentaciones }\end{array}$ & $\begin{array}{c}\text { Comprende } \\
\text { la visión }\end{array}$ & $\begin{array}{c}\text { Grupos } \\
\text { Artísticos } \\
\text { Contribución }\end{array}$ & $\begin{array}{c}\text { Orientación } \\
\text { al logro }\end{array}$ \\
\hline $\begin{array}{c}\text { Cambio } \\
\text { performance } \\
\text { MI }\end{array}$ & $\begin{array}{c}\text { Correlación } \\
\text { de Pearson } \\
\text { Sig. }\end{array}$ & 1 &, $100^{*}$ &, $102^{*}$ &, $214^{* *}$ &, $275^{* *}$ &, $162^{* *}$ &, $129^{* *}$ \\
(bilateral) \\
$\mathrm{N}$
\end{tabular}

* La correlación es significante al nivel 0,05 (bilateral).

** La correlación es significativa al nivel 0,01 (bilateral).

Fuente: Elaboración propia. 
difusión y de comercialización, como lo es el MICSUR. Además, se demuestra que el cambio de performance de los protagonistas o artistas consultados, está claramente relacionado con las oportunidades que logran conseguir en el mercado internacional, en tanto, se trata de integrar nuevos espacios o contextos culturales y dar respuesta a las necesidades del mercado. Todo esto demostrando, además, la posibilidad que tienen los representantes del sector cultural de diversificar y transformar sus servicios en pro de integrar nuevos espacios comerciales.

Respecto a los cargos que se encuentran distribuidos dentro de cada emprendimiento cultural, demuestran su relación con la decisión de variar el performance establecido para integrar el mercado internacional, de manera que, la estructura interna de cada caso manifiesta la articulación requerida con las necesidades a nivel global. Y siguiendo la internacionalización transfronteriza, en el caso latinoamericano, se logra el reconocimiento de este cambio de actuaciones de los emprendedores culturales, al tratar de responder a las necesidades del mercado y así ser tenidos en cuenta en nuevos mercados. El integrar nuevos elementos o generar variaciones asociadas a la inclusión de nuevas técnicas o miembros, determina su cambio de performance para la consecución de mayores oportunidades en el mercado internacional. De esta manera, se evidencia el elemento innovador dentro de los emprendimientos culturales en Latinoamérica. Por esto, y siguiendo a Howkins (2001), la innovación que se requiere en este sector mezcla los elementos creativos del ser humano y sus implicaciones en la economía, no como elementos separados, sino como dos herramientas que generan mayores posibilidades de acción y de consecución de entrada a nuevos mercados. El reconocer el mercado en el que están presentes y el entender la visión del mercado actual se relaciona con el cambio de performance que este tipo de emprendimientos genera con el fin de lograr ingresar al mercado internacional; así, se trata de la posibilidad de integrar un mercado ya estudiado y conocido, aprovechando esas características y esa visión que se realiza a partir de su presencia en el mismo. Se relaciona, de manera muy importante, el hecho del reconocimiento del trabajo en redes y el aporte que hacen otros grupos al cambio de su performance y la posibilidad de integrar el mercado internacional, demostrando que el sector cultural actúa bajo principios de trabajo colaborativo que incentiva su presencia en conjunto en escenarios globales. Según Johanson y Mattson (2015), las posibilidades de conseguir procesos de internacionalización en empresas, tiene que ver con el trabajo en redes, y este es uno de los elementos que se logró demostrar a partir del reconocimiento del sector cultural en Latinoamérica, frente a quienes comparten escenarios y espacios de difusión. La orientación que se le da a las acciones de cada emprendimiento de tipo cultural y la superación de estándares inicialmente marcados están relacionados con el cambio de performance que los llevaría al mercado internacional, demostrando el trabajo de cada grupo de artistas respecto 
a sus propias limitaciones. Por último, el reconocimiento y el grado de aplicabilidad o de cumplimiento de los objetivos de cada emprendedor o del grupo de emprendedores tiene una repercusión en la decisión de transformar su performance y así buscar la entrada al mercado internacional, lo que supone que al realizar estas acciones de trabajo en redes, las financian de manera propia, sin contar con el apoyo del Estado o de inversores privados, ya sea por desconocimiento de los convenios o por la dificultad de acceder a los mismos; razón por la cual, contar con el apoyo de nuevos actores que se integran a las redes de trabajo mediante el networking se vuelve motor de crecimiento para estos emprendimientos creativos.

\section{Conclusiones}

Es importante mencionar que, los datos y el mapeo de las industrias culturales y creativas en Latinoamérica, aún tiene una deuda pendiente, debido a que las mediciones más completas y desarrolladas por instituciones supranacionales no llegan a años actuales, que se trata de construcciones estatales que van poco a poco, que contemplan algunos sectores $\mathrm{y}$ que siguen en el trabajo de construcción de datos que permitan mejores resultados investigativos, más específicos $\mathrm{y}$ aplicables en más casos.

A partir del desarrollo de esta investigación, se logró identificar a los emprendimientos creativos como los conglomerados de acciones del sector cultural, cuyos objetivos están enfocados al éxito empresarial, al reconocimiento social y al establecimiento de nuevas oportunidades de negocio, dentro y fuera de su país de origen. Del mismo modo, pretenden vincular su producción artística con el contexto en el que se encuentran, mediante prácticas que logren impactar en el contexto en el que se ubica. Así también, la economía creativa representa la actividad del sector cultural, desde un punto de vista empresarial, debido a que se trata de la aglomeración de prácticas que buscan mejoras tanto económicas como de impacto social y que integran la dimensión social con la generación de riqueza.

La tipificación de las estrategias utilizadas por los emprendimientos creativos en Latinoamérica, están enfocadas en el establecimiento de relaciones, ya sean comerciales o de cooperación, pero siempre con fines de ampliación y crecimiento hacia nuevos mercados. Estas estrategias se definen desde la teoría de redes, entendida como la construcción de nuevas relaciones en nuevos mercados y la conexión a sistemas ya establecidos, de reciprocidades comerciales; así como el establecimiento de acuerdos de cooperación en la región.

Todo esto, teniendo en cuenta el alcance investigativo, el cual no pretende ser definitorio, ni exclusivo, sino más bien un punto de partida para futuras investigaciones que integren la totalidad de las variables propuestas, seleccionen muestras mayores, pero que, sobre todo, se de en escenarios interdisciplinares y vinculantes, que permitan un acercamiento más completo a lo que supone la 
internacionalización de las prácticas del sector cultural.

Los emprendimientos creativos de Latinoamérica, no se caracterizan por tener una estrategia de internacionalización claramente definida, pero utilizan mecanismos de conexión primaria y solitaria, si se quiere, para establecer alianzas que les permitan entrar en nuevos mercados, desde la difusión y el fortalecimiento de sus bienes y servicios, es decir, se pudo reconocer que los canales que poseen son desestructurados, que la internacionalización se realiza de forma particular o individual y que no se cuenta con el apoyo gubernamental completo. También que sus mecanismos están desconectados del funcionamiento del ecosistema creativo o del espacio en donde actores primarios y secundarios actúan en pro del sector.

Asimismo, la identificación a partir del método exploratorio, del networking como catalizador de oportunidades en mercados externos para las industrias culturales de Latinoamérica, es primordial para facilitar los procesos de comunicación, en tanto, logra un enfoque en el mensaje que quiere transmitirse, permite establecer un contacto directo con las personas y actores pertinentes y alcanza una dimensión de negocios mayor, en poco tiempo. Además, no exige niveles profesionales o laborales específicos y permite una unión de actores con objetivos comunes.

El trabajo en redes y el trabajo colaborativo se establecieron como la base de las estrategias de internacionalización de la economía creativa, ya sea de manera formal como espacio delimitado con la totalidad de actores del sistema, o de manera informal al reunir representantes de todo tipo en torno al sector cultural. Esto, debido a la importancia de la integración de nuevos actores que benefician los nuevos emprendimientos. Así, la apertura de espacios y el establecimiento de relaciones, se identificó como la principal herramienta utilizada por estas nuevas iniciativas como método de internacionalización. De esta manera, el análisis de las variables de pensamiento sistémico, permitió identificar las prioridades en torno a la aplicabilidad y reconocimiento del emprendimiento, frente al conjunto de la labor cultural, evidenciando que, si bien están interesados en los procesos de internacionalización, los que lo han logrado han utilizados alianzas estratégicas o han llegado a mercados externos mediante una financiación propia y alejada del trabajo en conjunto.

Igualmente, la cooperación transfronteriza, fue uno de los hallazgos más relevantes para el fortalecimiento de los procesos de internacionalización, en tanto permite la creación de nuevas redes de apoyo y crecimiento empresarial para los emprendimientos creativos. Teniendo en cuenta que para la región latinoamericana no existe una legislación específica para el fortalecimiento del sector cultural. Sin embargo, sí existen ciertas integraciones de bloques económicos que le han brindado un espacio a las industrias culturales que deben ser aprovechadas por quienes poseen este tipo de emprendimientos para 
lograr una ampliación de su mercado. Si bien se reconoce la intención y la identificación de mecanismos de internacionalización del sector cultural y creativo de la región, no existen bases fundamentadas y efectivas para la inserción en nuevos mercados externos, debido tanto al desconocimiento como a la falta de efectividad en las acciones realizadas, por lo que se resalta la necesidad y utilización apropiada de la teoría de redes. Por esta razón, se requiere un proceso de apropiación del trabajo en redes, como estrategia para los emprendimientos creativos, debido a que permite que las relaciones que se tejen entre los actores del sector cultural, alcancen un incremento de oportunidades empresariales y de difusión, que además dependerá de la información que cada participante aporte. Todo con el fin de formar relaciones nuevas en espacios nuevos, intensificar el trabajo ya establecido con socios o participantes existentes y la combinación del reconocimiento de los actores con una amplia colaboración voluntaria entre diferentes entidades, y la fuerza impulsora como interés común, para desarrollar habilidades, o para aprender (Akbar, 2014).

Por otra parte, el establecimiento del sistema de redes de cooperación, comercio o inserción en mercados externos, dentro del sector cultural, ha contado con el desarrollo de las habilidades que presenta el emprendimiento cultural, esto es, el conjunto de prácticas propias de los representantes creativos, debido a que facilita la construcción de redes de trabajo para la consecución de objetivos comunes. $\mathrm{Y}$ es que, dentro de las industrias culturales y creativas, la cooperación y el trabajo conjunto, es la máxima representación del networking, pero para el logro y fortalecimiento de la red, es necesario contar con las habilidades de un emprendedor cultural, y tener un panorama amplio sobre lo que se necesita de acuerdo a su contexto.

Se debe decir que la identificación de procesos de internacionalización de emprendimientos creativos en Latinoamérica, mostró la articulación entre procesos públicos en donde el Estado debe tener un papel preponderante al abrir y facilitar los espacios y apoyos necesarios, el establecimiento de redes de trabajo, de colaboración y de reconocimiento y las prácticas del emprendimiento cultural, de manera que, sean posibles y de manera eficaz, la entrada en nuevos mercados y la difusión de la industria, sin embargo, "no basta con su reconocimiento o apoyo, sino que hace falta su direccionamiento, es decir, no se pueden convertir en herramientas que sigan legitimando las diferencias sociales o la jerarquización económica, deben, por el contrario, romper paradigmas que materializan la segregación de la sociedad" (Redondo Méndez, 2018, p.60); siendo el aporte principal, precisamente, y teniendo en cuenta las limitaciones del muestreo, la identificación de este tipo de estrategias y canales de trabajo del sector cultural, que superan y se diferencian de los procesos establecidos en sectores tradicionales de la economía.

Por último, y de acuerdo al alcance 
investigativo, se sugieren tópicos $\mathrm{y}$ tendencias para la investigación en industrias culturales y creativas que permitan un mayor desarrollo de sus procesos de internacionalización, y que tomen como referencia espacios completos de representación del sector cultural; así, se habla de turismo cultural, mercado global cultural, arte popular tradicional, bibliometría cultural, economía cultural y políticas culturales (Rodríguez et al., 2020).

\section{Referencias}

Akbar, K. (2014). Networking for regional development: a case study. EuroMed Journal of Business, 9(2), 149-163.

Bernal, C. (2010). Metodología de la investigación: administración, economía, humanidades y ciencias sociales, tercera edición. Bogotá: Pearson Educación.

Cardozo, P., Chavarro, A., y Ramírez, C. (2007). Teorías de internacionalización. Panorama, 1(3). DOI: http://dx.doi.org/ 10.15765/pnrm.v1i3.264

CISAC. (2015). Tiempos de cultura. El primer mapa mundial de las industrias culturales $y$ creativas. EYGM Limited.

Consejo Andino de Ministros de Relaciones Exteriores. (2011). Vigésimo tercera reunión ordinaria. Decisión 760, Creación del Consejo Andino de Ministros de Cultura y de Culturas. Lima (Perú).

CULTURANDE. (2011). Decisión 11/96 del Consejo del Mercado Común.

Ghalioun, B. (1998). Globalización, deculturación y crisis de identidad. Afers Internacionals, (43-44), 107-118.

Hernández-Acosta, J. (2020). Emprendimiento creativo. San Juan: Inversión cultural.

Hernández-Acosta, J., Redondo, A., y Ospina, O. (2018). Industrias culturales y economía creativa en Latinoamérica. Desarrollo económico y social en la región. Bogotá: Editorial Uniagustiniana. DOI: https://doi.org/10.28970/9789585498143

Hernández, R., Fernández, C., y Baptista, M. (2014). Metodología de la investigación, Sexta edición. México: McGraw Hill.

Howkins, J. (2001). The Creative Economy. London: Penguin Books.

Johanson, J., y Mattson, LG. (2015). Internationalisation in industrial systems. A network approach. En: Forsgren M., Holm U., Johanson J. (eds) Knowledge, Networks and Power. Palgrave Macmillan, London. https://doi.org/10.1057/9781137508829_5

Lindsay, D. (2005). Cracking the Networking CODE: Four steps to priceless business relationships. Plano: World Gumbo Publishing.

MERCOSUR. (1996). Protocolo de Integración Cultural. Decisión 11/96 del Consejo del Mercado Común.

Ministerio de Cultura de Colombia. (2010). Compendio de Politicas culturales. Bogotá: Colombia.

Ortega-Mohedano, F., Jiménez-Sánchez, A., y Lavín, J. (2018). Industrias culturales y composición de los personajes en las series de animación infantil emitidas en España. Revista Latina de Comunicación Social, (73), 74-85.

PNUD. (2010). Economía creativa: una opción factible de desarrollo. Informe 2010. Naciones Unidas.

Ramírez, L. (2013). Japón y la internacionalización de su cultura como estrategia diplomática. Observatorio Iberoamericano de la 
Economía y la Sociedad del Japón, 5(18), 1-19.

Redondo Méndez, A.C. (2018). Industrias culturales latinoamericanas y su aporte al desarrollo social. En: J. Hernández Acosta, A. C. Redondo Méndez y O. Ospina Martínez (eds.) Industrias culturales y economía creativa en Latinoamérica. Desarrollo económico y social en la región (pp. 23-70). Bogotá: Editorial Uniagustiniana.

Rodríguez, L. (2018). Economía creativa en América Latina y el Caribe: mediciones y desafíos (Nota Técnica No IDB-TN-01488). Banco Interamericano de Desarrollo. DOI: http://dx.doi.org/10.18235/0001308

Rodríguez, L., Redondo, A., Ospina, O. y Pinzón, C.A. (2020). La innovación desde perspectivas investigativas de las industrias culturales y creativas. En: Sepúlveda, J. (ed) Estrategia y gestión organizacional (pp. 170-183). Medellín: Corporación Universitaria Americana.

Saucedo, H. (2020). La creatividad recurso económico: como estrategia de crecimiento. Investigación y Negocios, 13(21), 23-36. DOI: https:// doi.org/10.38147/inv\&neg.v13i21.81

Servicio de Inserción Profesional, Prácticas y Empleo-SIPPE. (2013). Guía práctica de Emprendimiento Social y Cultural. Gobierno de España, Ministerio de Educación, Cultura y Deporte. Universidad de Salamanca

UNESCO. (2009). Marco de Estadísticas Culturales (MEC) de la UNESCO 2009. Montreal: Instituto de Estadística de la UNESCO.

Yúdice, G. (2019). Políticas culturales y ciudadanía. Educ. Real, 44(4). DOI: https://doi.org/10.1590/2175-623689221 
